\title{
Evolución de la Microestructura para Diferentes Configuraciones de la Corriente Continua Pulsada en el Proceso de Soldadura GTAW Autógeno
}

\author{
Manuel Camus Maldonadoํㅜ (D) Víctor Vergara Díaz ${ }^{1}$ (D) \\ ${ }^{1}$ Universidad de Antofagasta, Departamento de Ingeniería Mecánica, Antofagasta, Chile.
}

Como citá: Maldonado MC, Díaz VV. Evolución de la Microestructura para diferentes configuraciones de la corriente continua pulsada en el proceso de soldadura GTAW autógeno. Soldagem \& Inspeção. 2020;25:e2535. https://doi.org/10.1590/0104-9224/SI25.35

\begin{abstract}
Resumen: El objetivo del estudio es verificar la influencia de la corriente doblemente pulsada, corriente pulsada y corriente continua constante sobre la microestructura en un acero inoxidable austenítico $316 \mathrm{~L}$ cuando se utiliza el proceso de soldadura GTAW autógeno. Para el desarrollo de este estudio de comparación todos los ensayos se realizaron con la misma energía de soldadura con el objetivo de verificar la influencia que presenta la modulación de la corriente sobre la microestructura de la región fundida. Los resultados indican que la corriente doblemente pulsada fue la que presentó el mayor refinamiento de la microestructura asociado a la mayor frecuencia de pulsación de la corriente alcanzada. Los perfiles de microdureza para los tres formatos de corriente utilizados revelan que existe un leve aumento del valor de dureza para el formato de corriente doblemente pulsada respecto al pulsado convencional y a la corriente continua constante. Esto se debe al mayor refinamiento de la microestructura que experimenta la zona de fusión debido a la mayor agitación de la pileta líquida cuando el proceso de soldadura opera con altas frecuencias de pulsación.
\end{abstract}

Palabras clave: 316L; GTAW autógeno; Refinamiento.

\section{Influence on Microstructure of the Autogenous GTAW Process for Different Modulations of the Pulsed Current}

\begin{abstract}
The purpose of this study is to verify the influence of double-pulsed current, pulsed and constant direct current on the microstructure of the $316 \mathrm{~L}$ stainless steel austenitic when the autogenous GTAW welding process is used. To carry out this comparison study, all the tests were executed with the same welding energy in order to verify the influence that presents the modulation of the current on the microstructure of the molted region. The results indicate that double-pulsed current was the one that presented the greatest refinement of the microstructure also associated with the higher frequency of pulsation of the reached current. The micro hardness profiles for the three currents formats used reveal that there is a slight increase in the hardness value for the double-pulsed current respect to the conventional pulsing and constant direct current. This is due to the greater agitation of the melted pool when the welding process operates at high pulsation frequencies.
\end{abstract}

Key-words: 316L; Autogenous GTAW; Refinement.

\section{Introducción}

El proceso GTAW (Gas Tungsten Arc Welding), se caracteriza por el empleo de un electrodo no consumible de tungsteno, el cual establece el arco eléctrico con la pieza a soldar. El proceso se acompaña con protección de un gas inerte (argón o helio), o una mezcla de ambos. El proceso se puede realizar utilizando diferentes modos de corriente denominados de corriente continua constante, corriente continua pulsada y corriente continua doblemente pulsada. En la soldadura con corriente continua pulsada, se tiene mejor control del arco y mejor estabilidad cuando se compara con la corriente continua c

onstante. Además, permite aplicar una gran intensidad de calor en la corriente de pulso que se traduce en una muy buena fusión y penetración, por otro lado, en el intervalo de corriente de base el arco se mantiene estable y permite que la zona de soldadura se enfríe y solidifique. La corriente continua pulsada se considera como un medio externo para generar refinamiento del grano en el metal solidificado en la soldadura. Esto toma importancia, considerando el hecho que la estructura del grano del metal de soldadura puede afectar significativamente las propiedades de este último. Cuando se utiliza corriente continua doblemente pulsada existe la imposición de dos niveles de corriente media (corriente de base térmica y corriente de pulso térmico), de esta manera dos niveles de energía. Con la imposición de la corriente de pulso existe una

Recibido: 22 Oct. 2019. Aprobado: 30 Sept. 2020

E-mail: manuel.camus@uantof.cl (MCM)

Este es un artículo publicado en acceso (Open Access) abierto bajo la licencia Creative Commons Attribution Non-Commercial, que permite su uso, distribución y reproducción en cualquier medio, sin restricciones siempre que sin fines comerciales y que el trabajo original sea debidamente citado. 
fusión más efectiva del material base y del metal de aporte y con la imposición de la corriente base un enfriamiento de la pileta fundida.

(Sharir et al., 1978) quien obtuvo refinamiento de grano usando el proceso de soldadura GTAW para unir placas de tantalio mediante arco pulsado. El refinamiento de grano obtenido por Sharir se explica en que la pileta líquida es subenfriada cuando el calor agregado se reduce drásticamente durante el ciclo de baja corriente en el proceso de soldadura con pulsación del arco. Esto sucede cuando la corriente alcanza su valor mínimo o intensidad de base, lo que produce nucleación en la superficie teniendo como consecuencia el refinamiento del grano.

Padmanaban y Balasubramanian (2011) en su trabajo sobre la influencia de los parámetros de la corriente pulsada en las propiedades mecánicas y metalúrgicas en aleaciones de magnesio AZ31B soldadas por el proceso GTAW con corriente pulsada, mostró que la alta dureza en la zona de fusión y la distribución uniforme de precipitados fueron las razones principales para que se generara una resistencia a la tracción mayor de dichas uniones, por lo que este tipo de procesos son importantes para aplicarse en la soldadura de aleaciones de titanio. Se puede reducir el crecimiento de grano en la zona afectada térmicamente y refinar la microestructura de la zona de fusión y con ello mitigar la reducción de las propiedades mecánicas y la posible pérdida de ductilidad. Farahani et al. (2012) en su trabajo sobre el proceso de soldadura GTAW con corriente continua constante y corriente continua pulsada en una aleación 617 divulgaron un refinamiento de la microestructura en la zona de fusión y el aumento de la resistencia al impacto al pasar de corriente continua constante a corriente continua pulsada.

Espinosa y Vergara (2015) en su trabajo caracterización del proceso de soldadura TIG cold wire con corriente doblemente pulsada divulgaron que para tres niveles de corriente media total ensayados (110, 120 y $130 \mathrm{~A}$ ), en la medida que se aumenta la corriente media existe un aumento en el ancho del cordón, disminuye el refuerzo y aumenta la penetración. Además, se observa del análisis de la microestructura en acero al carbono que para todas las configuraciones propuestas del formato de corriente, el predominio es de ferrita acicular y en la medida que se aumenta la corriente media total aparece ferrita Widmanstätten.

Raveendra y Kumar (2013) estudiaron los efectos de la corriente continua constante y la corriente continua pulsada (frecuencia de pulsación 5 y $10 \mathrm{~Hz}$ ) sobre un acero inoxidable 304. Los resultados encontrados revelan que existe un refinamiento de la microestructura, además variaciones en la magnitud de dureza presentando los mayores valores para corriente continua pulsada (frecuencia de pulsación $5 \mathrm{~Hz}$ ). Los menores valores de dureza corresponden a corriente continua constante debido a una microestructura más grosera. Los ensayos de tracción no presentan una diferencia significativa en las propiedades mecánicas para ambas modulaciones de corriente.

Cruz et al. (2016) estudiaron el efecto de la corriente pulsada en el proceso de soldadura GTAW en el material base (Titanio 6A14V), con y sin metal de aporte (Aporte: ERTi-5Eli). Los resultados encontrados revelan que las propiedades mecánicas de la unión son afectadas significativamente con respecto a la frecuencia, tanto utilizando metal de aporte o sin uso de este. En el caso de la soldadura con corriente pulsada, existe un aumento de aproximadamente $30 \mathrm{MPa}$ en la resistencia a la tracción, si se utiliza metal de aporte con respecto al autógeno. Se encontró que las probetas soldadas con corriente continua fracturaron en el cordón de soldadura, las probetas soldadas con corriente pulsada fracturaron en la zona afectada térmicamente, lo cual puede atribuirse a la diferencia entre la microestructura del metal base y de la ZAT. El ancho de cara y raíz disminuyó cuando ocurrió un cambio de corriente continua constante a corriente continua pulsada (frecuencia de pulsación $20 \mathrm{~Hz}$.), mientras que de 20 a $50 \mathrm{~Hz}$ no presentó un cambio significativo.

En los perfiles de dureza tanto en las probetas sin y con metal de aporte se observa que la dureza Vickers en el metal depositado con aporte es superior a la dureza en la misma región del metal fundido sin aporte.

Por tanto, el presente trabajo de investigación tiene por objetivo determinar la influencia de la corriente continua constante y diferentes modulaciones de corriente continua pulsada con 5 y $160 \mathrm{~Hz}$ de frecuencia de pulsación en el refinamiento de la microestructura, zona de fusión, usando un proceso de soldadura GTAW autógeno.

\section{Material y Procedimiento Experimental}

Para la realización de este estudio fue necesario el montaje de un banco de ensayos, en el Laboratorio de Procesos de Soldadura (LPS) del Departamento de Ingeniería Mecánica de la Universidad de Antofagasta. Dicho banco está compuesto por una máquina de soldar sinérgica MINITEC 200, para la traslación de la pistola se utilizó un sistema de avance lineal con un grado de libertad, lo que permite control y repetitividad de los parámetros de movimiento, abertura y finalización del arco. El monitoreo de la corriente de soldadura y de la tensión de arco fueron realizados con un sistema de adquisición propio de la fuente de soldadura, pistola comercial TIG con una capacidad de corriente de $200 \mathrm{~A}$, gas de protección como se muestra en la Figura 1. La máquina de soldar se desarrolló para operar en las opciones GTAW autógeno convencional y pulsado. Se mantuvo una distancia constante entre la punta del electrodo y el material base de $5 \mathrm{~mm}$. Se utilizó un electrodo de tungsteno no consumible EWth-2 de $2,4 \mathrm{~mm}$ de diámetro con un ángulo en la punta de $30^{\circ}$, gas de protección una mezcla $98 \% \mathrm{Ar}+2 \% \mathrm{H}_{2}$ con un caudal fijo de $12 \mathrm{l} \cdot \mathrm{min}^{-1}$ medido mediante flujómetro, velocidad de avance de la pistola $15 \mathrm{~cm} . \mathrm{min}^{-1}$. 


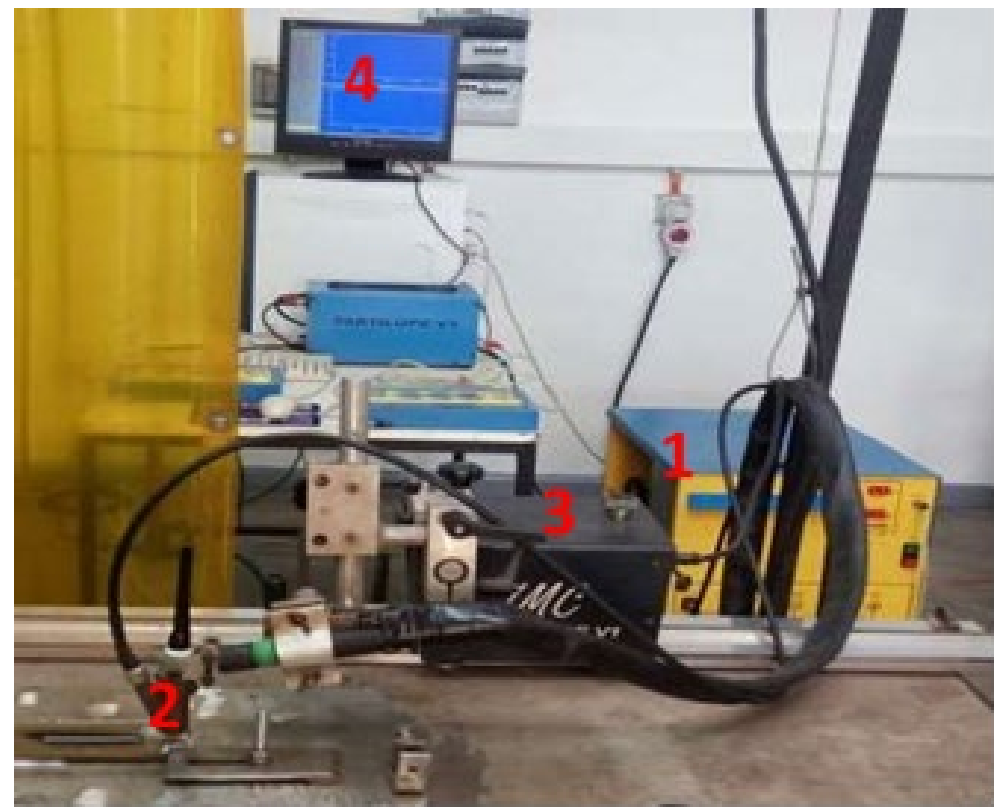

Figura 1. Banco de ensayos para proceso de soldadura GTAW montado en el Departamento de Ingeniería Mecánica UA. 1. Máquina de soldar; 2. Pistola adaptada TIG; 3. Manipulador de desplazamiento TARTILOPE; 4. Sistema de adquisición de datos (SAD).

Los ensayos se realizaron en la posición plana sobre chapas de acero inoxidable austenítico 316L de dimensiones $100 \times 150 \times 2,5 \mathrm{~mm}$, se extrajeron 3 cuerpos de prueba de dichas chapas. La composición química del metal base se evaluó mediante un equipo de espectrometría de emisión óptica de marca SPECTRO, modelo SPECTROLAB, según la norma ASTM E1086-14 (American Society for Testing and Materials, 2014). La Tabla 1 muestra la composición química del metal base acero inoxidable austenítico 316L. La Tabla 2 muestra la composición química del electrodo de tungsteno EWth2.

Tabla 1. Composición química acero inoxidable austenítico 316L.

\begin{tabular}{ccc}
\hline Elementos & $\begin{array}{c}\text { Norma ASTM A-240 (American Society for } \\
\text { Testing and Materials, 2000) } \\
0,03 \text { máx. }\end{array}$ & Muestra \\
\hline $\mathrm{C}$ & 2,0 máx. & 0,025 \\
\hline $\mathrm{Mn}$ & $\leq 0,75$ & 0,393 \\
\hline $\mathrm{Si}$ & $\leq 0,045$ & 0,014 \\
\hline $\mathrm{P}$ & $\leq 0,03$ & 0,0008 \\
\hline $\mathrm{S}$ & $16,0-18,0$ & 16,44 \\
\hline $\mathrm{Cr}$ & $2,0-3,0$ & 1,98 \\
\hline $\mathrm{Mo}$ & $10,0-14,0$ & 10,4 \\
\hline $\mathrm{Ni}$ & & 0,26 \\
\hline $\mathrm{Co}$ & & 0,267 \\
\hline $\mathrm{Cu}$ & & 0,189 \\
\hline $\mathrm{Nb}$ & & 0,0294
\end{tabular}

Tabla 2. Composición química electrodo de tungsteno EWth2.

\begin{tabular}{cc} 
Electrodo & EWth2 \\
Tungsteno & $97,30 \%$ \\
\hline Torio & $1,7-2,2 \%$ \\
\hline Otros óxidos & $0,50 \%$ \\
\hline
\end{tabular}

\subsection{Parámetros de soldadura}

Se realizaron ensayos de pruebas para determinar los parámetros de soldadura que permitieron obtener la matriz de experimentos necesarios para el estudio con el objetivo de verificar la influencia de la corriente continua constante y diferentes modulaciones de corriente continua pulsada sobre la microestructura. La Tabla 3 y 4 muestran las matrices de experimentos realizados tanto para corriente continua constante y corriente pulsada. 
Tabla 3. Plan de ensayos experimental utilizando corriente continua doblemente pulsada.

\begin{tabular}{cccccc}
\hline \multicolumn{2}{c}{ Pulso térmico (PT) } & & \multicolumn{3}{c}{ Base térmica (BT) } \\
Corriente de pulso & $\mathrm{I}_{\mathrm{p}} \mathrm{PT}$ & $160[\mathrm{~A}]$ & Corriente de pulso & $\mathrm{I}_{\mathrm{p}} \mathrm{BT}$ & $160[\mathrm{~A}]$ \\
\hline Corriente de base & $\mathrm{I}_{\mathrm{b}} \mathrm{PT}$ & $80[\mathrm{~A}]$ & Corriente de base & $\mathrm{I}_{\mathrm{b}} \mathrm{BT}$ & $120[\mathrm{~A}]$ \\
\hline Tiempo de pulso & $\mathrm{T}_{\mathrm{p}} \mathrm{PT}$ & $3[\mathrm{~ms}]$ & Tiempo de pulso & $\mathrm{T}_{\mathrm{p}} \mathrm{BT}$ & $3[\mathrm{~ms}]$ \\
\hline Tiempo de base & $\mathrm{T}_{\mathrm{b}} \mathrm{PT}$ & $3[\mathrm{~ms}]$ & Tiempo de base & $\mathrm{T}_{\mathrm{b}} \mathrm{BT}$ & $3[\mathrm{~ms}]$ \\
\hline Período & $\mathrm{TPT}$ & $0,1[\mathrm{seg}]$ & Período & $\mathrm{TBT}$ & $0,1[\mathrm{seg}]$ \\
\hline Corriente media (PT) & $\mathrm{I}_{\mathrm{m}} \mathrm{PT}$ & $120[\mathrm{~A}]$ & Corriente media (BT) & $\mathrm{I}_{\mathrm{m}} \mathrm{BT}$ & $140[\mathrm{~A}]$ \\
\hline Corriente media total & $\mathrm{I}_{\mathrm{m} T}$ & $130[\mathrm{~A}]$ & Potencia & & \multirow{2}{*}{$1841[\mathrm{~W}]$} \\
\hline
\end{tabular}

$\mathrm{I}_{\mathrm{p}} \mathrm{PT}$ : Corriente de pulso en el pulso térmico; $\mathrm{I}_{\mathrm{b}} \mathrm{PT}$ : Corriente de base en el pulso térmico; $\mathrm{I}_{\mathrm{p}} \mathrm{BT}$ : Corriente de pulso en la base térmica; $\mathrm{I}_{\mathrm{b}} \mathrm{BT}$ : Corriente de base en la base térmica; $\mathrm{T}_{\mathrm{p}} \mathrm{PT}$ : Tiempo de pulso en el pulso térmico; $\mathrm{T}_{\mathrm{b}} \mathrm{PT}$ : Tiempo de base en el pulso térmico; $\mathrm{T}_{\mathrm{p}} \mathrm{BT}$ : Tiempo de pulso en la base térmica; $\mathrm{T}_{\mathrm{b}} \mathrm{BT}$ : Tiempo de base en la base térmica; TPT: Período de pulso en el pulso térmico; $I_{\mathrm{m}} \mathrm{PT}$ : Corriente media en el pulso térmico; $I_{\mathrm{m}}$ : Coorriente media total; TBT: Período de pulso en la base térmica; $I_{m} B T$ : Corriente media en base térmica.

Tabla 4. Plan de ensayos experimental utilizando corriente continua constante y pulsada.

\begin{tabular}{|c|c|c|c|c|c|c|c|}
\hline Ensayo & $\operatorname{Ip}[\mathrm{A}]$ & Ib [A] & $\mathrm{tp}[\mathrm{ms}]$ & tb $[\mathrm{ms}]$ & $\operatorname{Im}[A]$ & $f[\mathrm{~Hz}]$ & Pot [W] \\
\hline $\mathrm{CCP}(\mathrm{EN})$ & 160 & 100 & 100 & 100 & 130 & 5 & 1946 \\
\hline $\operatorname{CCC}(\mathrm{EN})$ & \multicolumn{2}{|c|}{130} & \multicolumn{2}{|c|}{ NA } & 130 & NA & 1851 \\
\hline
\end{tabular}

CCP(EN): Corriente continua pulsada electrodo negative; CCC(EN): Corriente continua constante electrodo negative; Ip: Corriente de pulso; Ib: Corriente de base; tp: Tiempo de pulso; tb: Tiempo de base; f: Frecuencia de pulsación de corriente; Im: corriente media; Pot: Potencia; NA: No aplica.

\subsection{Metalografía}

Las probetas para el análisis metalográfico se extrajeron de los cuerpos de prueba, con la presencia de la zona de fusión (ZF), zona afectada térmicamente (ZAT) y metal base. La caracterización microestructural cuantitativa de las probetas procedió por medio de microscopia de luz (ZEISS- AXIOTECH) y un procesamiento digital de imágenes (KS-PHASE). Para estos efectos se procedió según la norma ASTM E-3 (American Society for Testing and Materials, 1995). La metalografía de las probetas se realizó mediante técnicas normales de lijado, para terminar con pulido en alúmina de 1,0; 0,3 y 0,05 $\mu$ m. Para revelar la microestructura a rasgos generales se utilizó un ataque electrolítico en una solución de ácido nítrico en agua destilada, bajo una tensión de 3 voltios y 5 segundos de inmersión.

Además, se cuantificó la fracción de ferrita en la zona de fusión mediante los diagramas de De Long y Schaeffler (Soldatelli Borsato, 2001), para ello se determinó el valor del níquel equivalente y cromo equivalente para ambos diagramas. También se utilizó la técnica de ferritoscopía para cuantificar la fase mencionada.

\subsection{Difracción de rayos $X$}

La identificación estructural de las fases componentes en la zona de fusión hizo uso de técnicas de difracción de rayos $\mathrm{X}$ para agregados policristalinos. Se utilizó un difractómetro SIEMENS D 5000 equipado con monocromador de grafito para el haz difractado, radiación Ka1 de cobre $(\lambda=0,15406 \mathrm{~nm} ; 40 \mathrm{KV}, 30 \mathrm{~mA})$, rendija de divergencia de $1 \mathrm{~mm}$, rendija de dispersión de $1 \mathrm{~mm}$ y rendija receptora de $0,6 \mathrm{~mm}$, geometría de Bragg-Brentano y modalidad de barrido $(\theta-\theta)$; el rango de barrido se fijó en 30 a $85^{\circ}$, con un paso de $0,02^{\circ}$ y tiempo de medición de 10 segundos por paso. El instrumento operó bajo el sistema de programas DIFFRAC-Plus para adquisición y procesamiento de los datos experimentales.

\subsection{Propiedades mecánicas}

La cuantificación del valor de microdureza se realizó en un microdurómetro Zwick Roell modelo ZHV $\mu$, utilizando un indentador de diamante y una carga de 1 gramo, las mediciones se realizaron cada 0,5 $\mathrm{mm}$ en la zona de fusión.

\section{Resultados y Discusiones}

Las Figuras 2 a la 4 muestran las macrografías y los oscilogramas de corriente y tensión de arco en la zona de fusión realizada con corriente continua pulsada para frecuencias de pulsación de 5 y 160 Hertz y corriente continua constante. 

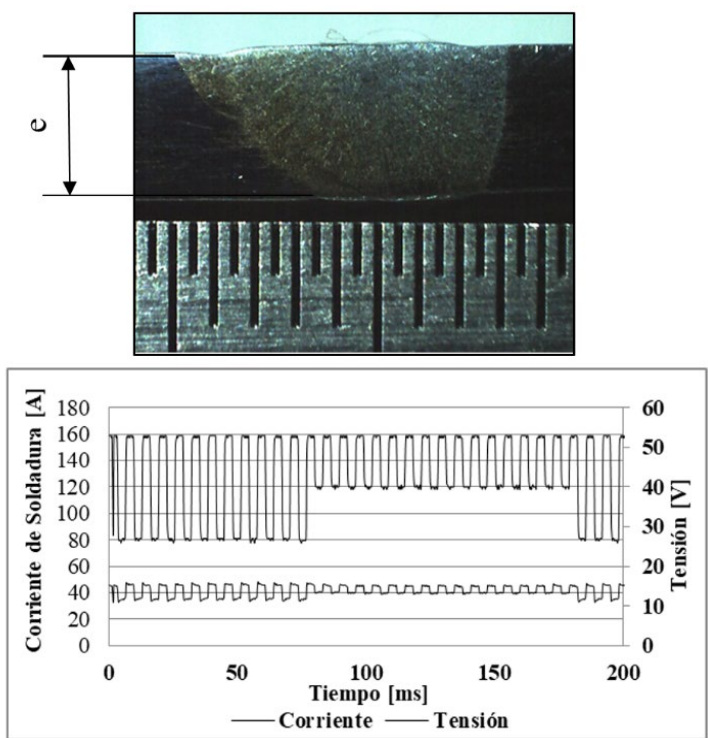

Figura 2. Macrografía y oscilograma de corriente y tensión para un formato de corriente continua doblemente pulsada $Q_{1}=7,634\left(\mathrm{~kJ} . \mathrm{cm}^{-1}\right)$.
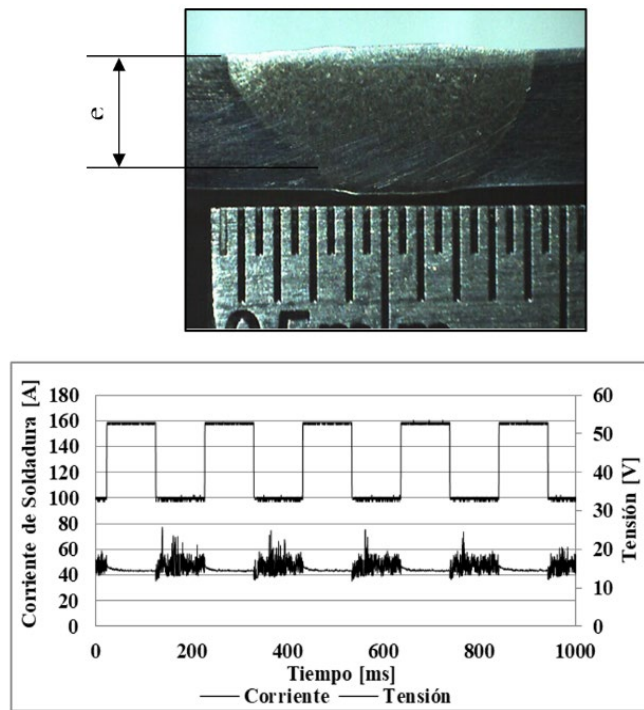

Figura 3. Macrografía y oscilograma de corriente y tensión para un formato de corriente pulsada $\mathrm{Q}_{2}=7,784\left(\mathrm{~kJ} . \mathrm{cm}^{-1}\right)$.
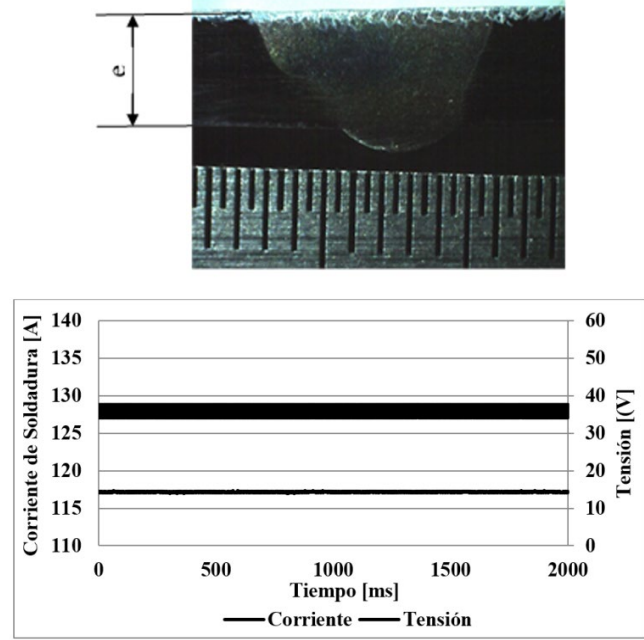

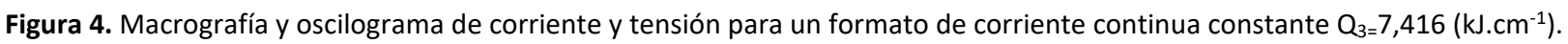


La Figura 5 muestra las magnitudes para el ancho de la zona de fusión para las diferentes modulaciones de corriente. No se observa una variación significativa, aunque el menor valor de $8,18 \mathrm{~mm}$ se presenta para el proceso realizado mediante corriente continua doblemente pulsada, el mayor valor de $8,7 \mathrm{~mm}$ se presenta para corriente continua constante (Qi et al., 2013). La Figura 6 muestra los valores de la energía impuesta para las diferentes modulaciones de corriente, todos los ensayos de realizaron con la misma energía impuesta, de tal modo, verificar la influencia que presenta la modulación de la corriente en la microestructura de la zona de fusión.

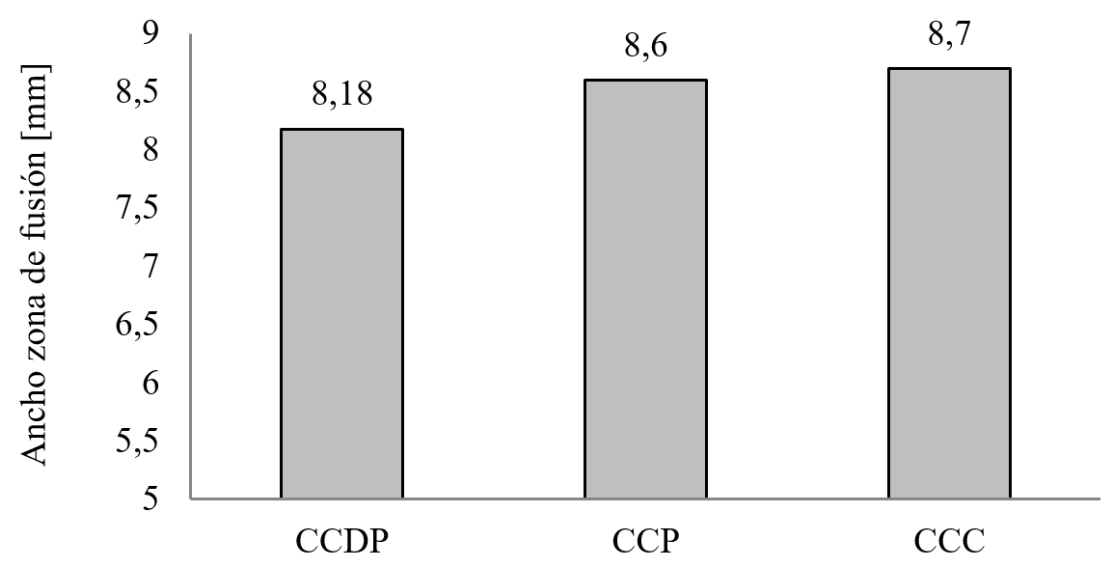

Modulaciones de Corriente

Figura 5. Ancho de la zona de fusión para las diferentes modulaciones de corriente. CCDP: Corriente continua doblemente pulsada; CCP: Corriente continua pulsada; CCC: Corriente continua constante.

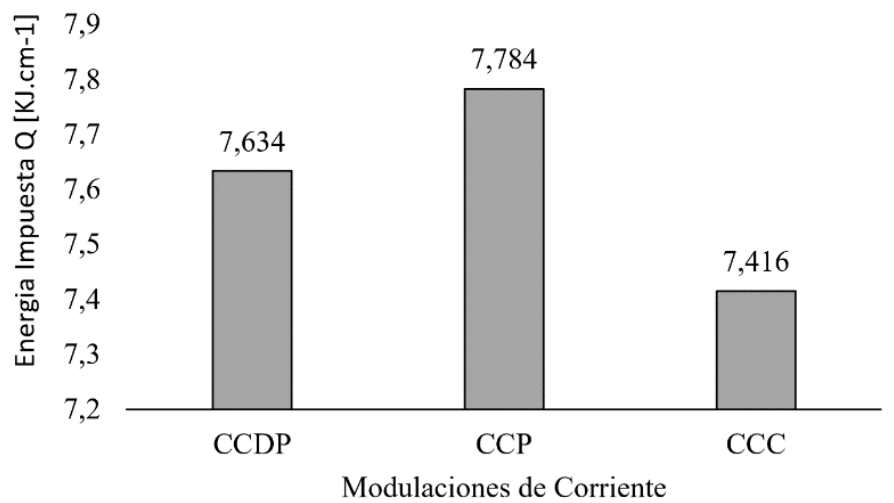

Figura 6. Energía impuesta para las diferentes modulaciones de corriente. CCDP: Corriente continua doblemente pulsada; CCP: Corriente continua pulsada; CCC: Corriente continua constante.

\subsection{Cuantificación de ferrita en la zona de fusión}

La cuantificación de la fase ferrita presente en la zona de fusión se realizó mediante los diagramas de Schaeffler y De Long para ello se determinó el valor del níquel equivalente y cromo equivalente para ambos diagramas.

Diagrama de Schaeffler (1)

$C r_{\text {equiv. }}=19,02 N i_{\text {equis. }}=11,81$

Diagrama de De Long (2)

$C r_{\text {equis. }}=19,02 N i_{\text {equis. }}=14,8$

La Tabla 5 muestra la cuantificación de la fase ferrita $\delta$ y ausentita en la zona de fusión por medio de los diagramas de Schaeffler, De Long, microscopia cuantitativa, ferritoscopía y técnicas de difracción de rayos $X$ (Programa computacional TOPAS III basado en el método Ríetveld). Los valores más exactos corresponden a la técnica de difracción de rayos $\mathrm{X}$ mediante un barrido por pasos. 
Tabla 5. Valores de las fracciones de ferrita $\delta$ y austenita en la zona de fusión.

\begin{tabular}{cccc}
\hline Técnicas de cuantificación & Formato de corriente & Fe $\gamma$ [\%] & Fe $\delta$ [\%] \\
Diagrama Schaeffler & & 95 a 90 & 5 a 10 \\
Diagrama De Long & CCC & ---- & 6,36 \\
Microscopia cuantitativa & CCP & 92,76 & 7,1 \\
& CCDP & 92,1 & 7,91 \\
Difracción Rayos X & CCC & 93,9 & 6,1 \\
& CCP & 93,4 & 6,6 \\
Ferritoscopía & CCDP & 92,4 & 7,7 \\
& CCC & 91,17 & 8,83 \\
& CCP & 91,9 & 8,2 \\
\hline
\end{tabular}

CCC: Corriente continua constante; CCP: Corriente continua pulsada; CCDP: Corriente continua doblemente pulsada.

\subsection{Difracción de rayos $X$}

Las Figuras 7 a 9 muestran los difractógramas de la zona de fusión realizado mediante el proceso de soldadura GTAW con corriente continua pulsada (frecuencias de pulsación 5 y $160 \mathrm{~Hz}$ ) y corriente continua constante. Se observan las líneas de difracción para ambas fases, siendo los máximos de difracción para la austenita y ferrita (111)y y (110) $\delta$ respectivamente. Además, se observa en la Figura 7 una orientación preferencial de los planos de austenita $\{220\}$, con respecto a los $\{111\}$, lo que indica una texturización del tipo $\{110\}$ inducida por la solidificación de la pileta líquida. El mismo fenómeno se presenta para corriente continuo constante siendo la orientación preferencial de los planos de austenita $\{200\}$, con respecto a los $\{111\}$. La fase ferrita $\delta$ no presenta el mismo fenómeno. Se puede afirmar que el proceso de soldadura no induce ninguna transformación de fases.

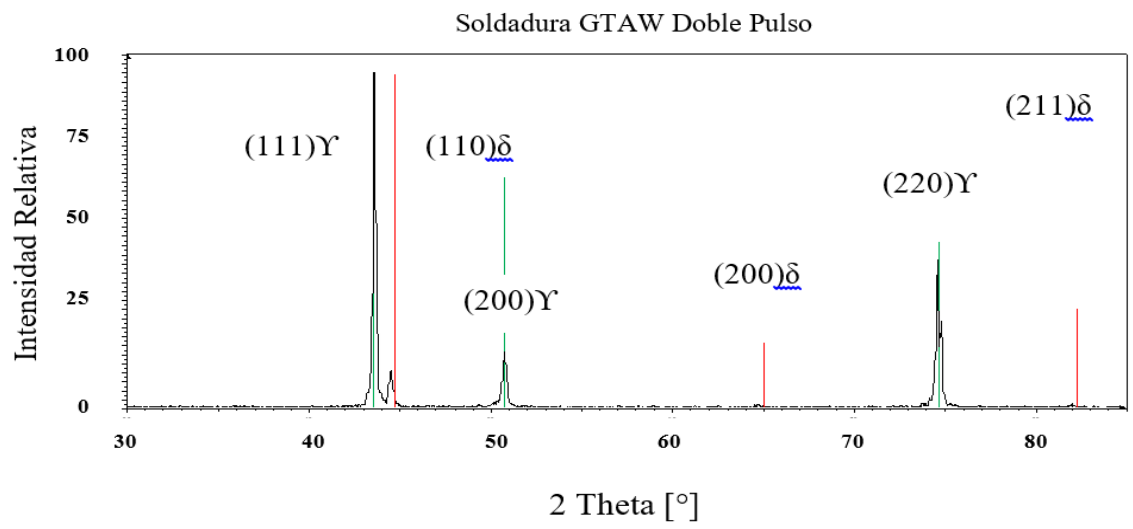

Figura 7. Difractógrama en zona de fusión proceso de soldadura GTAW doble pulso.

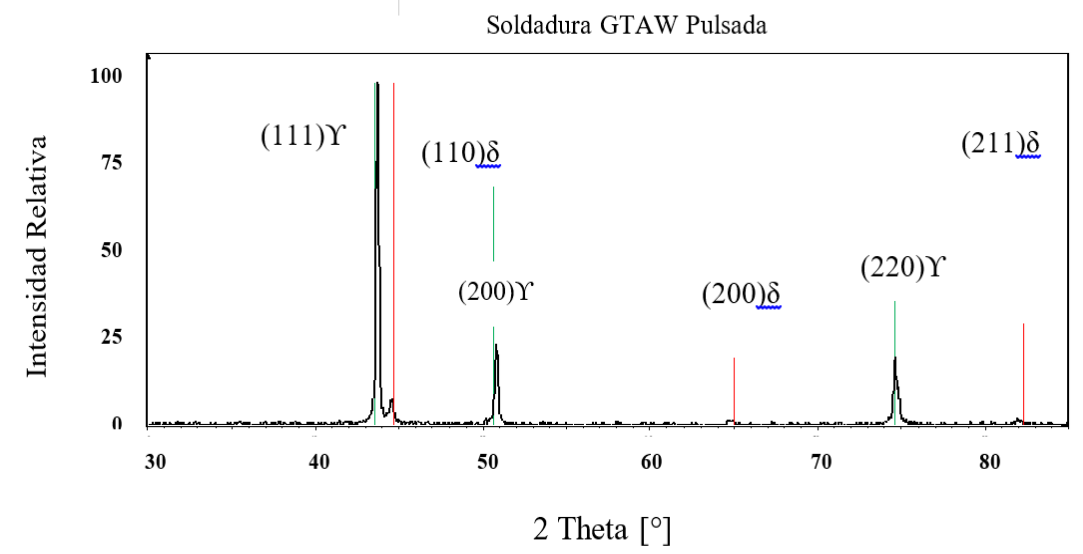

Figura 8. Difractógrama en zona de fusión proceso GTAW pulsado. 


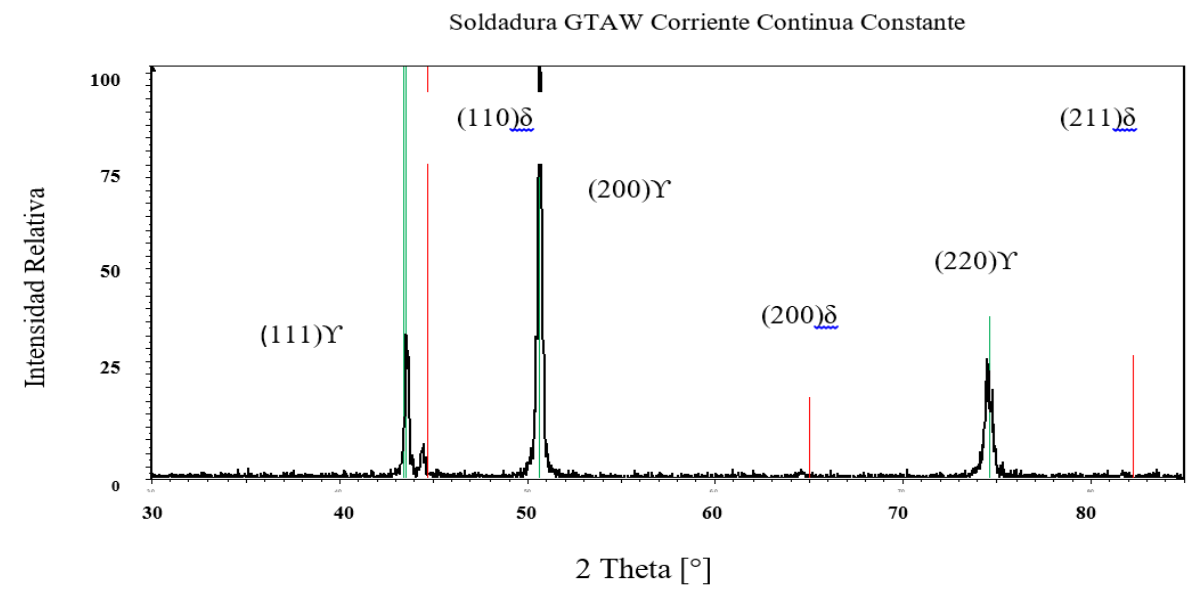

Figura 9. Difractógrama en zona de fusión proceso de soldadura GTAW mediante corriente continua constante.

En la Figura 10 se puede apreciar la evolución de la intensidad relativa de los tres primeros peaks correspondiente a la fase austenítica en estudio, donde se observa la intensidad relativa en los planos $\{111\},\{200\}$ y $\{220\}$ para el proceso de soldadura GTAW autógeno mediante corriente continua doble pulsada, corriente continua pulsada y corriente continua constante. Se ha agregado los peaks de una muestra de austenita teórica que se usa como patrón. Se observa que para las tres configuraciones de corriente se presenta textura siendo para corriente continua constante mayor, dándose en los tres planos analizado.

Esa orientación preferencial puede deberse a que el proceso de solidificación, se privilegian ciertas direcciones de crecimiento que puede deberse a:

1. Una mayor velocidad de crecimiento en las orientaciones $\{220\}$ y $\{200\}$ respecto de $\{111\}$;

2. Una mayor extracción de calor coincidente en esas orientaciones.

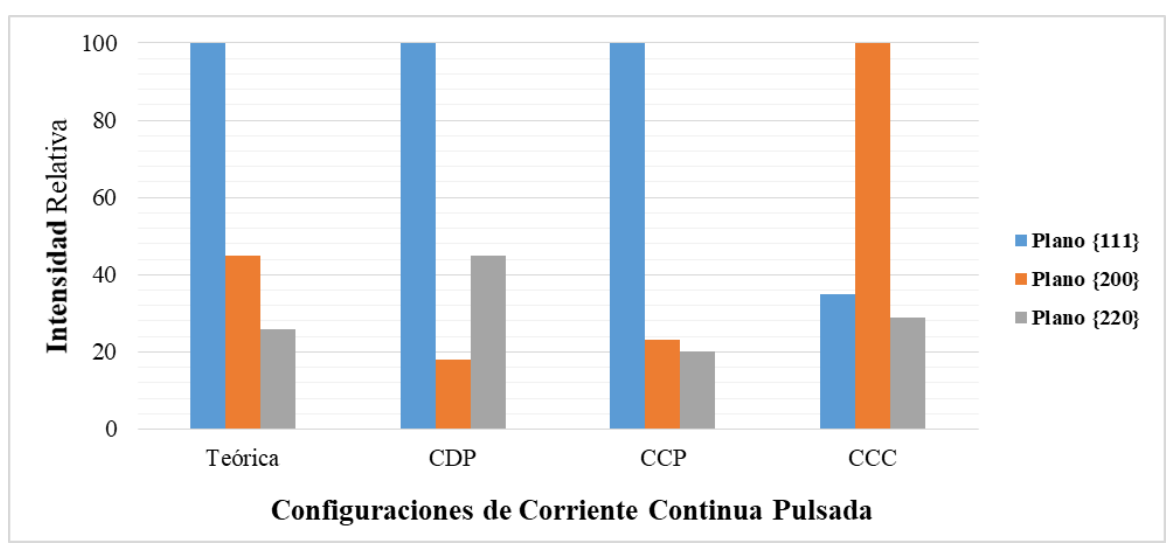

Figura 10. Evolución de la textura de la fase austenítica para diferentes configuraciones de corriente mediante el proceso de soldadura GTAW autógeno.

Al formarse la pileta líquida e iniciar su avance por la chapa de acero inoxidable 316L ésta genera un frente de gradientes de temperaturas los cuales aumentan conforme el metal fundido se desplaza longitudinalmente sobre la chapa. El calor de aporte se concentra en el centro de la pileta líquida y se mantiene a alta temperatura durante su desplazamiento longitudinal gracias al flujo de metal fundido de alta temperatura proveniente de los lados frontal y posterior de la fuente. La solidificación de la pileta líquida se produce en la dirección de la soldadura.

\subsection{Análisis metalográfico}

La zona de fusión presenta una microestructura bifásica constituida de dendritas de austenita (zona clara) en una matriz ferrítica (zona oscura) como se muestra en la Figura 11. Se observa un leve refinamiento de la microestructura para el proceso GTAW mediante corriente doblemente pulsada (alta frecuencia de pulsación $160 \mathrm{~Hz}$ ), respecto al pulsado convencional y a la corriente continua constante. En el pulsado térmico existe la imposición de dos niveles de corriente media (corriente de base térmica y corriente de pulso térmico), de esta manera dos niveles de energía. Con la imposición de la corriente de pulso existe 
una fusión más efectiva del material base y del metal de aporte y con la imposición de la corriente de base existe un enfriamiento de la pileta líquida, induciendo mejores condiciones de tensión superficial y viscosidad garantizando de esta manera un control sobre la pileta líquida (Rodrigues, 2003).

Elevadas frecuencias de pulsación producen mayor agitación de la pileta líquida provocando la fragmentación de las dendritas. Esta fragmentación de las dendritas conduce a la formación de una estructura más fina y conlleva a una mayor resistencia y dureza de la zona de fusión o unión soldada (Hadadzadeh et al., 2014). A diferencia con el proceso de corriente continua constante, en el modo pulsado la energía térmica necesaria para fundir el material base se suministra solamente durante los pulsos de corriente de pulso durante breves intervalos de tiempo permitiendo que el calor se disipe en el material base. Como consecuencia disminuye el calor de aporte en la soldadura, resultando en una zona afectada térmicamente (ZAT) más estrecha con un reducido esfuerzo residual y distorsión (Wang et al., 2006; Senthil Kumar et al., 2007).

La corriente continua pulsada conduce a una solidificación controlada, lo que a su vez induce a una estructura dendrítica más fina y a un menor engrosamiento del grano en la zona afectada térmicamente tanto en el modo autógeno como con alambre de aporte (Manikandan et al., 2014). El proceso de soldadura GTAW con corriente continua involucra mayor calor de aporte en contraste con la corriente continua pulsada que utiliza un calor de aporte controlado.
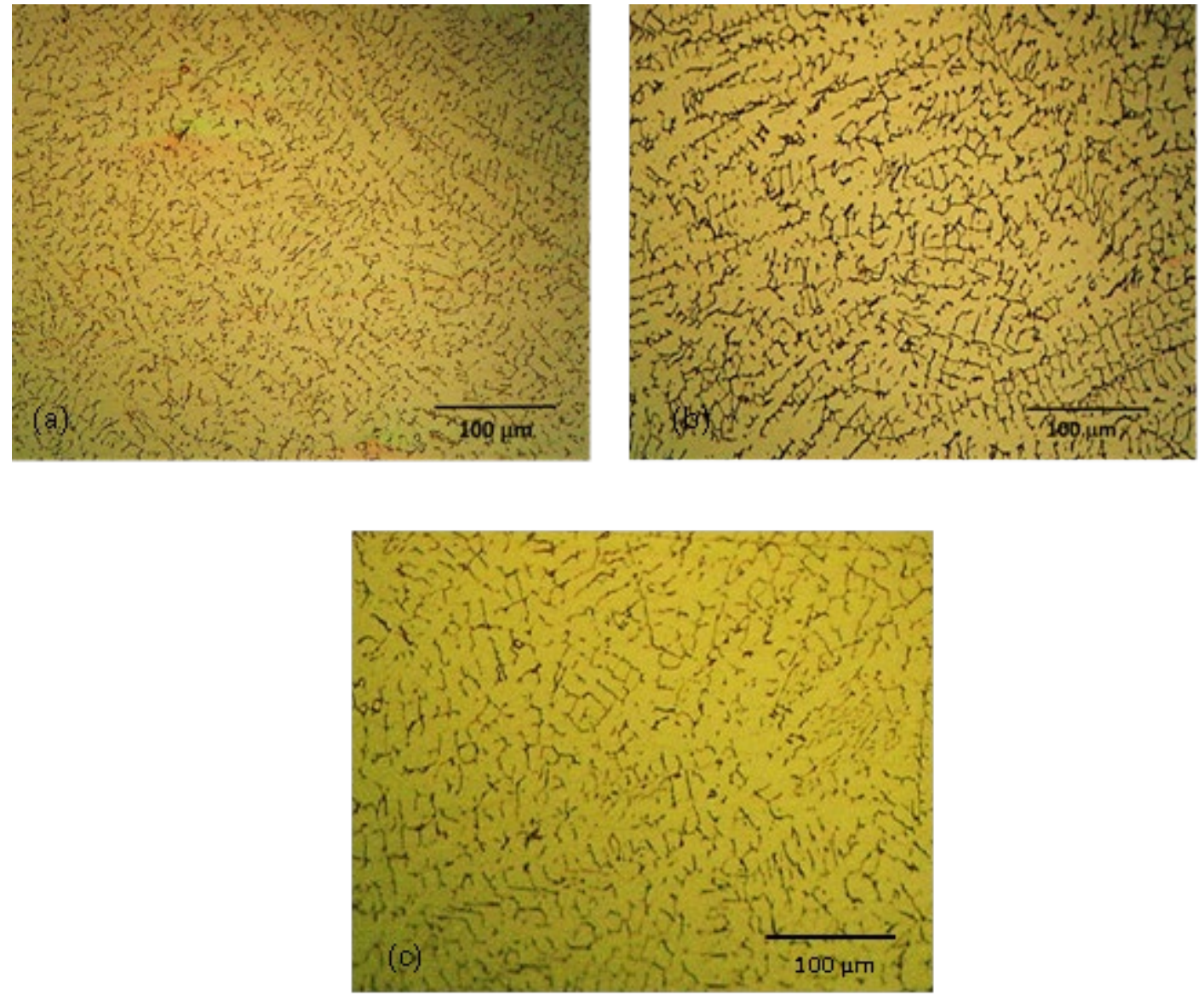

Figura 11. Microestructura zona de fusión para diferentes modalidades de corriente: (a) Corriente continua doblemente pulsada;

(b) Corriente continua pulsada y (c) Corriente continua constante.

\subsection{Perfil de dureza}

La Figura 12 muestra el perfil de microdureza para los tres formatos de corriente utilizados en el proceso GTAW autógeno. Se observa un leve aumento del valor de dureza para el formato de corriente doblemente pulsada (alrededor de 10 puntos) respecto al pulsado convencional y a la corriente continua constante. Esto se debe al mayor refinamiento de la microestructura que experimente la zona de fusión debido a la mayor agitación de la pileta fundida, lo que conlleva, a un aumento de la velocidad de enfriamiento y la fragmentación de las dendritas. La posición de las identaciones en los cuerpos de prueba se relizaron en regiones similares. 


\section{Microdureza Hv}

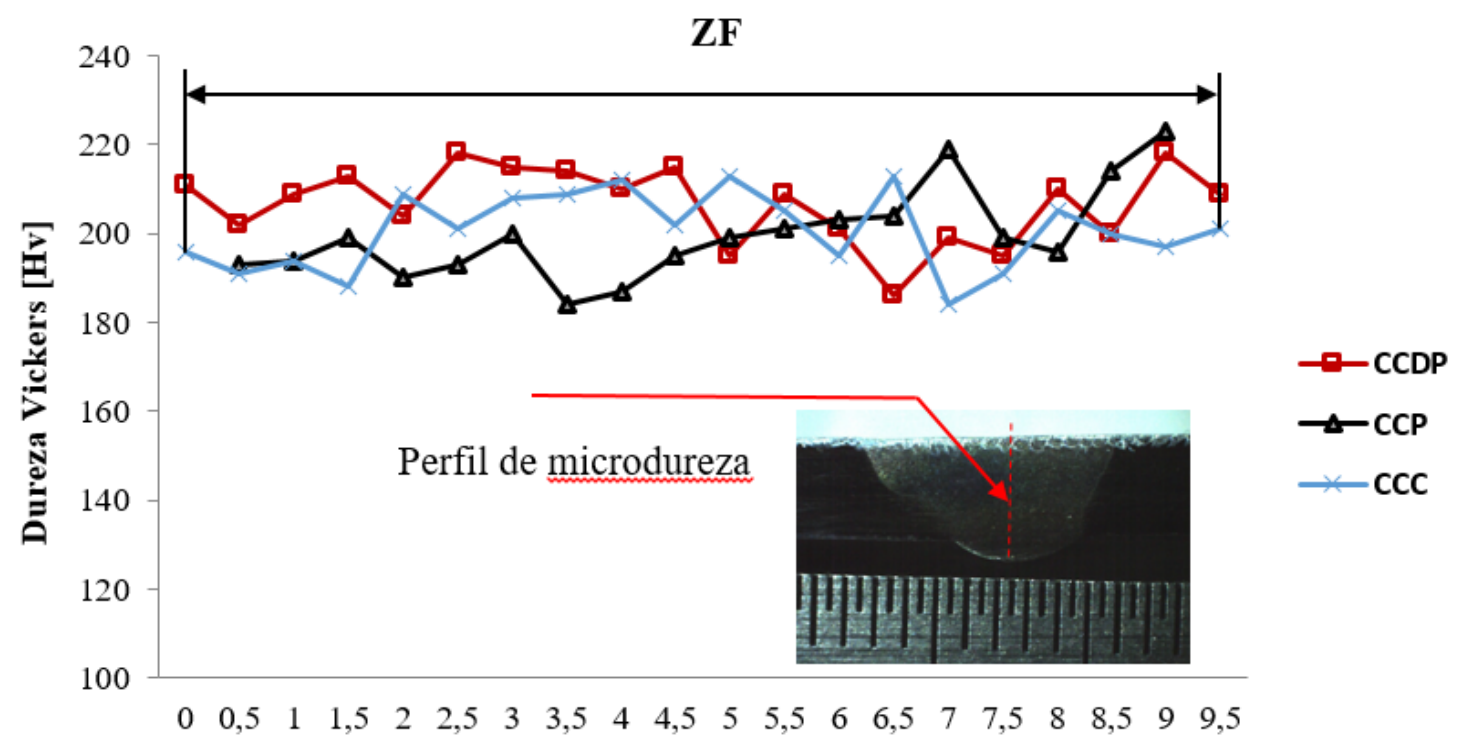

Distancia [mm]

Figura 12. Perfil de dureza en la zona de fusión proceso GTAW autógeno para los diferentes formatos de corriente. CCDP: Corriente continua doblemente pulsada; CCP: Corriente continua pulsada; CCC: Corriente continua constant; ZF: Zona Fundida.

\section{Conclusiones}

Con base a los resultados presentados en este trabajo, se destacan las siguientes conclusiones:

(a) El ancho de la zona de fusión disminuye con altos niveles de frecuencia de pulsación (Proceso GTAW autógeno utilizando corriente doblemente pulsada $160 \mathrm{~Hz}$.);

(b) La zona de fusión para las tres modulaciones de corriente presenta orientación preferencial de las líneas $\{200\}$ y $\{220\}$ de la austenita respecto a la línea $\{111\}$. El fenómeno no se presenta en la fase ferrítica;

(c) No se observa precipitación de fase durante el proceso de soldadura en la zona de fusión para las tres modulaciones de corriente;

(d) Se observa un leve refinamiento de la microestructura en la zona de fusión para corriente doblemente pulsada en comparación a la corriente pulsada y corriente continua constante debido al alto nivel de frecuencia de pulsación $(160 \mathrm{~Hz})$;

(e) Un leve incremento del valor de microdureza presentó el formato de corriente doblemente pulsado respecto al pulsado convencional y a la corriente continua constante.

\section{Referencias}

American Society for Testing and Materials. Standard specification for heat-resisting chromium and chromium-nickel stainless steel plate, sheet, and strip for pressure vessels, ASTM A240/A 240M - 00. ASTM International, 2000, 1-11

American Society for Testing and Materials. Standard specification for preparation of metallographic specimens, ASTM E3-95. ASTM Standards. 1995;03(01):1-8.

American Society for Testing and Materials. Standard test method for analysis of austenite stainless steel by spark atomic emission spectrometry, ASTM E1086-14. ASTM International, 2014, 1-6

Cruz, C., Gala, H., Mosquera, J., Gámez, H. Efecto de la corriente pulsada en el proceso de soldadura GTAW en titanio 6A14V con y sin metal de aporte. Revista de Metalurgia. 2016;53(3):e071. http://dx.doi.org/10.3989/revmetalm.071.

Espinosa A, Vergara V. Caracterización del proceso de soldadura TIG cold wire con corriente doblemente pulsada. In: Associação Brasileira de Soldagem. Congresso Nacional de Soldagem - CONSOLDA XLI; 2015 October 12-15; Salvador, Brazil. São Paulo: ABS; 2015.

Farahani E, Shamanian M, Ashrafizadeh F. A comparative study on direct and pulsed current gas tungsten arc welding of alloy 627. AMAE International Journal on Manufacturing and Materials Science. 2012;2(1):1-6. 
Hadadzadeh A, Ghaznavi MM, Kokabi AH. The effect of gas tungsten arc welding and pulsed-gas tungsten arc welding process parameters on the heat affected zone-softening behavior of strain-hardened Al-6,7Mg alloy. Materials \& Design. 2014;55:335-342. http://dx.doi.org/10.1016/j.matdes.2013.09.061.

Manikandan M, Arivazhagan N, Nageswara Rao M, Reddy GM. Microstructure and mechanical properties of Alloy C-276 weldments fabricated by continuos and pulsed current gas tungsten arc welding techniques. Journal of Manufacturing Processes. 2014;16(4):563572. http://dx.doi.org/10.1016/j.jmapro.2014.08.002.

Padmanaban, G., Balasubramanian, V. Influences of pulsed current parameters on mechanical and metallurgical properties of gas tungsten arc welded AZ31B magnesium alloy. Metals and Materials International. 2011;17(4):679-687.

Qi BJ, Yang MX, Cong BQ, Liu FJ. The effect of arc behavior on weld geometry by high-frequency pulse GTAW process with Cr18Ni9Ti stainless steel. International Journal of Advanced Manufacturing Technology. 2013;66(9-12):1545-1553. http://dx.doi.org/10.1007/s00170-0124438-z.

Raveendra A, Kumar BVR. Experimental study on pulsed and nonpulsed current TIG welding of stainless steel sheet (SS304). International Journal of Innovative Research in Science, Engineering and Technology, 2013;2(6):2337-2344.

Rodrigues S. Influência do processo MIG/MAG térmico sobre a microestrutura e geometría da zona fundida [tese doutorado]. Florianópolis: Programa de Pos-gradução em Engenharia Mecânica, Universidade Federal de Santa Catarina; 2003.

Senthil Kumar T, Balasubramanian V, Sanavullah MY. Influences of pulsed current tungsten inert gas welding parameters on the tensile properties of AA6061 aluminium alloy. Materials \& Design. 2007;28(7):2080-2092. http://dx.doi.org/10.1016/j.matdes.2006.05.027.

Sharir Y, Pelleg J, Grill A. Effect arc vibration and current pulses on microstructure and mechanical properties of TIG tantalum welds. Metals Technology. 1978;V5(1):190-196. http://dx.doi.org/10.1179/mt.1978.5.1.190.

Soldatelli Borsato K. Caracterização microstructural e de propiedades de chapas espessas de aço inoxidavel duplex UNS S31803, sometido a ciclos térmicos de soldagem. [tese doutorado]. Florianópolis: Programa de Pos-graduação em Engenharia Mecânica, Universidade Federal de Santa Catarina; 2001

Wang SH, Chiu PK, Yang JR, Fang J. Gamma $(\gamma)$ phase transformation in pulsed GTAW weld metal of duplex stainless steel. Materials Science and Engineering A. 2006;420(1-2):26-33. http://dx.doi.org/10.1016/j.msea.2006.01.028. 